\title{
Les didactiques au centre Formation genevoise des enseignants du secondaire ${ }^{1}$
}

\section{Bernard Schneuwly, Charles Heimberg et Rémy Villemin}

Parmi les multiples dispositifs de formation à l'enseignement secondaire qui cohabitent au sein de la Confédération suisse, cet article présente et interroge celui nouvellement mis en place dans le canton de Genève qui donne aux didactiques disciplinaires une place centrale. Les raisons et les hypothèses qui sous-tendent ce "pari " des didactiques sont questionnées à partir de trois approches complémentaires: la première s'intéresse au contexte genevois, tentant de cerner l'effet du contexte local sur l'adaptation de mouvements de réformes d'envergure nationale et internationale. La deuxième analyse la nature des savoirs dispensés durant la formation en se focalisant sur l'articulation des didactiques disciplinaires avec les savoirs scientifiques, avec les savoirs issus des sciences de l'éducation et avec les savoirs issus de la pratique professionnelle. Enfin, la troisième porte sur l'inscription des didactiques dans un dispositif de formation universitaire interfacultaire qui articule recherche scientifique et formation à l'enseignement.

La réforme de la formation des enseignants en Suisse, entreprise depuis les années 90, est décrite sous les labels de «tertiarisation, scientificisation, autonomisation et harmonisation" (Criblez, 2010). Ces transformations institutionnelles se manifestent à travers quelques éléments-clé, constatés à une large échelle internationale, et qu'on peut, avec Tardif et Borges, définir comme:

a) l'élévation, l'allongement et l'universitarisation de la formation; b) l'intégration de la recherche dans la formation et la construction d'une base de connaissances (knowledge base) ou d'un référentiel de compétences propre au travail enseignant; c) la place centrale réservée à la formation pratique et aux stages; d) la collaboration entre les institutions de formation et les milieux scolaires; e) les modes de contrôle des apprentissages et des niveaux de compétences. (2009, p. 110)

Ces transformations ont lieu dans le cadre d'un processus de professionnalisation qui opère sur la longue durée et comprend de multiples facettes, dont précisément celui de la formation (Novoa, 1998). Pour ce qui concerne cette 
dernière, ce processus implique toujours que les savoirs élaborés par la profession sont systématiquement reconstruits sous forme explicite dans des institutions spécialisées (Hofstetter \& Schneuwly, 2002) où ils sont, selon la belle formule de Bourdoncle (1991, 1993), "professés» à l'intérieur de champs disciplinaires. Ce rapport est particulièrement complexe pour la profession enseignante pour laquelle des savoirs ne sont pas seulement outils de travail - comme pour les médecins par exemple -, mais également objet: enseigner (en-signer selon l'étymologie) consiste précisément à donner l'accès aux savoirs par des signes (Hofstetter \& Schneuwly, 2009). La profession enseignante est la seule pour laquelle les savoirs ont ce double statut: savoirs à enseigner et savoir pour enseigner. Elle entretient par conséquent un rapport particulièrement complexe avec les disciplines académiques.

La profession d'enseignant secondaire se distingue du primaire par le fait que son principal champ disciplinaire de référence n'est pas professionnel. En effet, comme le montrent Schneuwly et Hofstetter (2006), les savoirs qui légitiment son statut sont construits en premier - ce terme a une valeur aussi bien génétique que logique - dans des disciplines qui ne sont pas orientées vers la pratique de l'enseignement, mais qui suivent une logique disciplinaire propre: mathématiques, littérature, histoire, etc.

Dans le contexte de tertiarisation des formations à l'enseignant entreprise dès les années 1990 en Suisse et au niveau international (Criblez, 2010), Genève réforme sa formation des enseignants du secondaire, donnant une forme locale spécifique aux rapports entre profession et discipline en donnant aux didactiques des disciplines une place centrale: telle est l'hypothèse développée dans le présent texte. Avec cette hypothèse, nous nous situons d'emblée dans un paradigme interprétatif qui, contrairement à des visions qui insistent sur les similitudes dans les mouvements internationaux de réformes éducatives, met l'accent sur les adaptations qu'implique tout transfert d'un modèle - ici celui de la tertiarisation au sens défini en introduction - dans un contexte local avec des pratiques culturelles spécifiques, historiquement constituées. Ni copie mécanique, ni déviance par rapport à un modèle, la réalisation concrète, localement spécifique, de réformes qui correspondent à des mouvements internationaux ${ }^{2}$, leur donne sens en articulant, de manière toujours originale, l'ancien et le nouveau (Schriewer, 2001). Cette articulation constitue la condition même de leur possibilité, le global n'existant que sous forme locale.

Dans cette perspective et à travers une étude de cas retraçant l'histoire de la réforme de la formation des enseignants du secondaire à Genève dans les années 2000, le présent texte explore, en se référant à des textes prescriptifs (concepts de formation, plans d'études, contenus annoncés des cours), le dispositif mis en place qui vise à transformer les rapports entre la profession d'enseignant du secondaire et les champs disciplinaires auxquels elle se réfere (Hofstetter \& Schneuwly, 2002; Lussi Borer, 2008). Pourquoi les didactiques ont-elles pris cette place importante dans le dispositif de formation des enseignants du secon- 
daire? Quel rôle jouent-elles dans la professionnalisation et la tertiarisation des formations à l'enseignement? Quels liens entretiennent-elles avec les savoirs académiques de référence des branches scolaires, avec les sciences de l'éducation, avec les savoirs professionnels des enseignants? Quelle place ont-elles dans un dispositif universitaire interfacultaire et comment s'organise leur rattachement aux différents champs disciplinaires? Telles sont les questions qu'abordera cet article en prenant pour objet de description et d'analyse les textes de référence de la formation et non pas sa réalisation concrète, ce qui aurait demandé une étude empirique prématurée après deux ans de fonctionnement.

Les sources utilisées pour décrire la réforme de la formation des enseignants du secondaire dans le contexte genevois proviennent d'une part d'investigations historiennes conséquentes dans les archives genevoises ${ }^{3}$, d'autre part de rapports, règlements et plans d'études produits dans le cadre de la réforme de la formation des enseignants du secondaire ${ }^{4}$. L'objectif du présent texte n'est ni de montrer le fonctionnement réel du dispositif, soumis à l'évidence à de nombreuses tensions et contradictions, ni, encore moins, de procéder à son évaluation. Il s'agit de mettre à la disposition des chercheurs une présentation d'un dispositif de formation qui a certaines particularités par rapport à d'autres, tout en le situant dans le mouvement général de réforme et de comprendre certaines raisons d'être de ces particularités par les conditions locales de son émergence. La référence à des sources officielles pour cet objectif peut se justifier en se référant, mutatis mutandis, à l'argumentation de Hébrard (1988): il s'agit de "textes prudents qui tiennent largement compte des possibilités d'innovation des professionnels et des structures [... qui] aiment souligner les continuités plutôt que les ruptures [...] les processus complexes d'élaboration de cette littérature garantissent d'une certaine manière sa validité» (p. 115).

Pour discuter du dispositif de formation préconisé et pour comprendre les raisons d'être de la place accordée aux didactiques, l'article s'appuie sur les travaux menés par l'Equipe de Recherche en Histoire des Sciences de l'éducation (ERHISE) de l'Université de Genève portant sur le conditionnement réciproque entre le développement des sciences de l'éducation et celui des formations à l'enseignement (Hofstetter \& Schneuwly, 2002; Hofstetter et al., 2007; Nóvoa, 1998; Tenorth, 1999) dans une perspective d'histoire et de sociologie des sciences (sociales) (Blanckaert, Blondiaux, Loty, Renneville \& Richard, 1999; Bourdieu, 2001; Pestre, 2006). Il convoque également une ample revue de littérature sur la nature et la place des savoirs en formation (Hofstetter \& Schneuwly, 2009) et notamment sur celle des savoirs didactiques (Hudson \& Schneuwly, 2007; Schneuwly, 2008).

L'article est construit en deux parties. Dans la première, nous précisons le contexte historique et culturel dans lequel la réforme se déploie. Puis nous décrivons la place et la nature des savoirs inscrits dans le dispositif genevois réformé de formation des enseignants du secondaire en mettant l'accent sur les savoirs didactiques. En conclusion, nous questionnons brièvement l'inscription 
institutionnelle du dispositif choisi en le mettant en perspective avec d'autres se situant dans le même mouvement de réforme, mais fonctionnant selon des modalités contrastées.

\section{Le contexte genevois}

Comme en de nombreuses autres contrées, la nécessité d'une formation professionnelle pour les enseignants secondaires ne va pas de soi. A Genève, les raisons qui ont poussé à sa mise en place au sein des facultés disciplinaires durant le premier $20^{\mathrm{e}}$ siècle sont «l'affaiblissement croissant de l'homologie entre savoirs professés [à l'université] et savoirs à enseigner dû à la différenciation croissante des disciplines académiques et scolaires ainsi que l'augmentation de l'hétérogénéité du corps enseignant secondaire découlant de la diversification du système secondaire» (Hofstetter, Schneuwly, Lussi \& Cicchini, 2004, p. 305). Dès la Seconde guerre mondiale, le dispositif change: la formation professionnelle est "extra-universitarisée» et confiée à la profession elle-même: les enseignants sont formés en emploi, par un pair selon un système de compagnonnage, sous le contrôle de l'administration.

\section{Un statut unique des enseignants du secondaire}

Dans le canton de Genève, le statut spécifique d'enseignant du secondaire inférieur n'a jamais existé pour différentes raisons historiques, notamment un haut niveau de qualification des enseignants du primaire - semi-universitaire depuis les années 30 -, un bassin de recrutement relativement large des enseignants du secondaire en quête d'emploi, une évolution particulière de ce qui est appelé aujourd'hui "école secondaire» (Berthoud, 2006; Hofstetter, 2010). Ce sont les professeurs du gymnase qui, tôt déjà, enseignent également dans d'autres écoles (école de commerce, école de culture générale, cycle d'orientation). Tous les enseignants du secondaire appartiennent donc à la même catégorie de personnel, sont payés de la même manière, ont le même cahier des charges, enseignent le même nombre d'heures et, surtout, suivent la même formation. Cela permet une mobilité des enseignants au sein des différentes écoles de niveau secondaire, une mobilité fortement défendue par les syndicats et associations professionnelles.

\section{Cinquante ans de formation professionnelle en compagnonnage}

Comme nous l'avons déjà dit, à partir des années 1940, la formation professionnelle genevoise des enseignants du secondaire ne se déroule plus simultanément avec les études universitaires disciplinaires. Les futurs enseignants étudient d'abord une ou plusieurs disciplines dites «enseignables» jusqu'au niveau de la licence, puis suivent une formation professionnelle. Le diplôme universitaire 
constitue le droit d'entrée dans l'enseignement en tant que maître en formation qui suit, en emploi, un cursus de formation constitué de deux composantes:

- une formation pratique comme enseignant en pleine responsabilité avec un accompagnement par un pair. Ces pairs sont des enseignants chevronnés, partiellement détachés de l'enseignement pour ce faire durant une période limitée - donc nullement des spécialistes de la formation -, qui accompagnent les nouveaux enseignants durant leurs deux premières années de pratique;

- des cours et séminaires, essentiellement de psychologie et de pédagogie.

D'abord organisée par les directions des écoles du secondaire en interaction avec l'Université, la formation est confiée dès 1965 à une institution spécialisée de formation des enseignants du secondaire, les Etudes pédagogiques de l'enseignement secondaire (EPES), qui prend en charge la totalité de la formation. La collaboration avec l'Université devient sporadique. Comment comprendre le choix de ce dispositif de formation? Celui-ci sert d'abord la volonté de l'administration scolaire et des directions d'établissement de prendre la main sur la formation à la profession, en contrôlant le processus de qualification professionnelle. Il donne également à la profession, à travers le système du compagnonnage, plus de poids dans la formation et la socialisation des futurs enseignants (Extermann, 2007). Enfin, il promulgue que l'activité professionnelle s'apprend essentiellement par le faire, toute prétention à sa théorisation suscitant une forte méfiance, la spécificité et l'expertise de l'enseignant du secondaire résidant dans sa maîtrise des contenus à enseigner et non pas dans sa connaissance théorique et technique des processus d'apprentissage chez les élèves ou d'enseignement chez l'enseignant (Schneuwly \& Hofstetter, 2006). C'est notamment sur ce dernier point que les enseignants du secondaire s'appuient pour se démarquer de ceux du primaire, dont la formation professionnelle se déroule pour partie à l'Institut universitaire des sciences de l'éducation. Les disciplines de référence de la profession des enseignants secondaires sont donc quasi exclusivement celles dans lesquelles ces derniers ont été formés à l'université (lettres, histoire, mathématiques, physique, géographie, etc.) et qui correspondent aux branches scolaires qu'ils enseignent. Les sciences de l'éducation, pourtant fortement développées par ailleurs à Genève en lien avec la formation des enseignants du primaire, n’y interviennent que marginalement.

Dans le sillage des réformes des formations à l'enseignement des années 1990, les EPES disparaissent pour laisser place en 1999 à un Institut de formation des maîtres et maîtresses du secondaire (IFMES). Toujours rattaché à l'enseignement secondaire, cet institut prolonge le dispositif précédent, tout en renforçant l'encadrement par les pairs et la formation théorique. Les ateliers donnés par les formateurs de référence deviennent notamment des ateliers de didactique, et non plus de méthodologie.» 
Une réforme basée sur un consensus cantonal dans un contexte défini au niveau suisse

Le concept même de cet Institut, géré par l'employeur, est rapidement mis en question. Dès 2003, la nécessité de répondre aux exigences de tertiarisation portées par les autorités suisses et européennes est ressentie par tous les acteurs: la profession, les directions d'école et du département de l'instruction publique, les associations professionnelles, les formateurs eux-mêmes, mais également l'Université qui, après plus de 50 ans d'absence, souhaite participer à nouveau à la formation professionnelle des enseignants du secondaire. ${ }^{5}$ Il s'agit dès lors de trouver des solutions aux problèmes définis comme suit:

- préparer pour l'enseignement au secondaire I et au secondaire II;

- baser la formation sur une alternance théorie-pratique à travers une formation en emploi;

- associer les enseignants en fonction à la formation;

- intégrer dans le dispositif de formation les principes de la Déclaration de Bologne;

- rapprocher le dispositif de formation des enseignants du secondaire du canton de Genève de celui de formation en hautes écoles tel qu'il est prévu dans l'ordonnance de la Conférence des directeurs de l'instruction publique (CDIP), à savoir un diplôme combiné nécessitant une formation scientifique de niveau master et une formation professionnelle de 94 crédits ECTS.

Ces contraintes institutionnelles vont donner à la formation des enseignants du secondaire un visage particulier que nous allons présenter en mettant au centre la question du rapport aux disciplines scientifiques.

\section{Quels savoirs pour former des enseignants du secondaire? Vers un nouveau rapport entre profession et disciplines}

Comme nous l'avons vu, la formation professionnelle et la construction de l'identité d'enseignant ne vont pas de soi dans l'enseignement secondaire: tant la prégnance de la discipline académique dans laquelle les enseignants sont initialement formés que la difficulté à considérer qu'enseigner peut et doit être l'objet d'une formation font obstacles. La nouvelle formation mise en place à Genève tente de transformer le rapport entre profession et disciplines en proposant un nouveau dispositif institutionnel de formation - un institut interfacultaire - et une articulation - notamment par l'entremise des didactiques disciplinaires entre trois types de "savoirs objectivés» (Barbier, 1996, p. 9) présents dans la formation (savoirs disciplinaires de référence, savoirs à enseigner et savoirs pour enseigner) qu'il s'agit de mettre également en relation avec des savoirs issus de la pratique de la profession. Nous décrivons cette tendancielle redéfinition du rapport entre profession et disciplines à travers quatre lieux d'articulation essen- 
tiels: l'institution qui met en rapport les disciplines; l'interrelation des disciplines elles-mêmes; les modalités de mise en rapport entre pratique et disciplines; l'intégration de la recherche dans la formation.

\section{Un institut interfacultaire, lieu d'articulation entre disciplines}

A l'intérieur d'une université, la formation des enseignants peut être institutionnalisée de deux manières: soit elle est prise en charge par une Faculté, comme par exemple au Québec ou aux États-Unis d'Amérique, comme c'était à ce moment déjà le cas pour la formation des enseignants du primaire à Genève sous la responsabilité de la Section des sciences de l'éducation; soit elle est assumée par une institution spécialisée transversale, interfacultaire. C'est cette dernière solution qui a été choisie à Genève. Quatre facultés portent l'Institut universitaire de formation des enseignants (IUFE) dont l'existence repose sur un règlement adopté par les Conseils participatifs de chacune d'elles et qui est en charge de toutes les formations d'enseignants. Ces quatre facultés sont impliquées dans la formation des enseignants soit dans les didactiques des branches d'enseignement (Facultés des lettres, des sciences et des sciences économiques et sociales), soit dans la formation professionnelle (Faculté de psychologie et des sciences de l'éducation). Elles participent à la supervision de l'Institut par le Conseil de l'IUFE (en règle générale, le doyen de chaque Faculté y siège), et à la gestion des filières de formation par l'Assemblée de l'IUFE qui nomme les comités de programme et adopte les plans d'études et règlements. ${ }^{6}$

La configuration interfacultaire de l'institution matérialise le double rapport constitutif de la profession enseignante aux savoirs: les savoirs à enseigner qui ont, au niveau secondaire, comme référence principale ${ }^{7}$ les savoirs parfois dits «savants», élaborés et transmis dans des disciplines étudiées dans le cursus de formation en Sciences, Lettres ou Sciences économiques et sociales, et les savoirs pour enseigner, systématisés et travaillés tout particulièrement dans le champ disciplinaire des sciences de l'éducation (Hofstetter \& Schneuwly, 2009). L'IUFE met donc institutionnellement en lien des savoirs disciplinaires divers, au cœur de la profession, grâce au fait qu'il est l'émanation de facultés spécialisées dans la production de ces savoirs et que son mode de fonctionnement implique ces facultés.

Ce dispositif institutionnel est complété par un autre au niveau du personnel enseignant. En effet, à l'Université de Genève, tous les professeurs officiant au sein d'instituts interfacultaires sont engagés par et administrativement insérés dans les Facultés. Ils constituent ainsi des «courroies de transmissions» mettant en rapport IUFE et facultés. Tous en effet enseignent aussi bien dans leurs facultés de référence (au moins un tiers temps) qu'à l'IUFE et participent activement aux instances des deux institutions. L'articulation entre disciplines - disciplines de référence, didactiques, sciences de l'éducation - est ainsi prévue tant au niveau des personnes qu'au niveau géographique, tous les enseignants se trouvant réunis sur un seul site afin de favoriser la circulation d'informations. 
Pluridisciplinarité, avec les didactiques au centre Si l'IUFE genevois est conçu selon des principes originaux dans le contexte suisse, il est soumis, comme d'autres institutions, aux règlements de reconnaissance élaborés par la CDIP qui régissent tous les instituts de formation suisses. L'architecture du programme pour la formation des enseignants du secondaire reprend donc les trois domaines de formation édictés par la CDIP: les didactiques, les sciences de l'éducation et la formation pratique professionnelle. Les modalités selon lesquelles sont conçus ces trois domaines prennent cependant une coloration locale particulière.

La didactique, qui a pour objet de recherche et de formation l'enseignement et l'apprentissage de savoirs organisés en discipline, comprend deux volets distincts, fortement articulés:

- formation pratique dans des ateliers liés aux stages;

- introduction aux concepts de base et à la recherche de la didactique comme discipline académique.

Conférer aux didactiques dans leur double fonction de réflexion pratique et de discipline de recherche une place centrale constitue un choix essentiel du dispositif genevois qui le distingue d'autres dispositifs. Ce choix a trois raisons d'être:

- il est d'abord basé sur la conception même de l'enseignement et de l'école avec au centre la création des conditions d'accès aux savoirs pour le plus grand nombre dans le sens large français du terme qui correspond assez bien au scire latin et qui inclut Wissen et Können (pour un développement de cette idée, voir Chevallard qui théorise cette question à travers sa notion de praxéologies, 1997; voir Schneuwly, 2011);

- ce positionnement est renforcé dans le contexte d'une formation qui s'adresse à un public hautement qualifié dans une ou plusieurs disciplines qui sont des références pour les branches scolaires. Ceci permet et nécessite à la fois une réflexion sur le découpage scolaire des savoirs proposés dans ces branches. La nécessité de cette réflexion est dictée autant, sinon plus, par les besoins d'enseignement en fonction des apprentissages et de la progression des élèves que par la logique propre de l'objet de savoir. En effet, la distance entre savoirs acquis par les étudiants et savoirs à enseigner est d'autant plus grande que la spécialisation est plus poussée. Les processus de transposition didactique doivent dès lors être étudiés avec minutie pour maîtriser les savoirs à enseigner.

- L'enseignant du secondaire est d'abord spécialiste de l'enseignement d'une discipline dont la didactique peut être considérée comme le savoir professionnel principal de référence: savoir pratique incarné dans les plans d'études et moyens d'enseignement, mais également savoir systématisé à propos des pratiques, que ce savoir soit praxéologique - comment enseigner? - ou descriptif et explicatif - comment fonctionne l'enseignement? 
La formation en sciences de l'éducation, quant à elle, réunit trois types d'interventions, portant à la fois sur des éléments de l'acte d'enseignement, sur le contexte social et scolaire de l'enseignement ainsi que sur l'identité professionnelle:

- des cours grand public dans quatre domaines centraux, donnés par des professeurs de l'université: histoire des systèmes scolaires; évaluation et sélection; pluralité des conditions sociales de genre et de cultures; développement et apprentissage en contexte scolaire;

- une unité de formation introduisant à la "profession enseignante». Quatre domaines y sont abordés: construction de la profession enseignante et de ses savoirs constitutif; postures de l'enseignant vis-à-vis des élèves; dimensions didactiques du travail enseignant; valeurs orientant la profession enseignante; - des ateliers portant sur des dimensions transversales de l'enseignement (rapport au savoir, gestion de la classe, participation à la vie de l'établissement), sur les MITIC, et sur ce que signifie «enseigner à des adolescents».

Ce dispositif pluridisciplinaire avec les deux composantes «didactique» et «sciences de l'éducation» est une manière de traduire le rapport complexe qu'entretient la profession enseignante avec les savoirs, pris ici dans le sens de savoirs explicites (pour une discussion, voir Hofstetter \& Schneuwly, 2009) ${ }^{8}$. Trois ensembles peuvent être distingués:

- des savoirs de référence qui constituent une part des savoirs à enseigner et dont l'appropriation se fait à travers la formation disciplinaire; notons que ces savoirs ne constituent nullement la totalité des références des savoirs à enseigner qui, eux, sont le résultat de processus complexes de transposition didactique;

- des savoirs à enseigner couplés à des savoirs pour les enseigner qui constituent l'objet des didactiques;

- des savoirs pour enseigner, ensemble complexes représentés par une partie des domaines des sciences de l'éducation.

La réorganisation de la formation dans un dispositif qui articule d'une manière nouvelle les disciplines contributives a des répercussions durables dans l'organisation universitaire elle-même et dans le développement des disciplines. On peut supposer que les changements dans les disciplines de référence académiques sont minimes 9 . Sans subir de changements fondamentaux parce que déjà développés dans le cadre de l'intégration de la formation des enseignants du primaire, les domaines des sciences de l'éducation sont renforcés pour assumer la charge supplémentaire de formation et se diversifient pour mieux inclure les réalités de l'enseignement secondaire. Les didactiques, elles, se trouvent profondément réorganisées: diversifiées, distribuées autrement dans l'organisation universitaire, elles sont dorénavant présentes non plus seulement en Section des sciences de l'éducation au sein de la Faculté de psychologie et des sciences de l'éducation, mais également dans les autres facultés, voire dans les autres Hautes Écoles (HEM et HEAD). 
Le renforcement de la place des didactiques n'est nullement spécifique à l'évolution genevoise. L'universitarisation de la formation des enseignants du secondaire, de manière générale, provoque une consolidation et un développement du champ des didactiques que l'on peut observer dans le contexte de la transformation de la formation des enseignants (pour la Suisse, voir Thévenaz \& Sieber, 2005; plus généralement pour l'Europe, Hudson \& Meinert, 2011). Le processus a cependant été mené de manière de manière particulière dans le contexte genevois pour plusieurs raisons:

- un longue tradition didactique y existe depuis fort longtemps, d'abord sous forme de psychopédagogie issue des travaux piagétiens (Schneuwly, 2008), puis transformée et renforcée notamment lors de l'intégration de la formation des enseignants du primaire en 1996 (Lussi \& Maulini, 2007);

- la participation des différentes facultés a permis une différenciation des lieux d'insertion des didactiques en les situant parfois proches des disciplines de référence, parfois proches des sciences de l'éducation, créant des ponts entre ces deux lieux;

- l'insertion universitaire donne la possibilité de créer une relève scientifique à travers la réalisation de doctorats dans les différentes didactiques disciplinaires, un prérequis pour le développement d'un champ disciplinaire.

Ces conditions propices au développement des didactiques permettent d'entrevoir, dans le microcosme genevois, les contours d'un champ disciplinaire appelé de leurs voux par les autorités suisses (Ambühl \& Stadelmann, 2010). Notons cependant que des mécanismes institutionnels sont nécessaires pour garantir ce champ: des dérives dans d'autres universités ont incité les acteurs de la réforme genevoise à introduire des mécanismes de contrôle empêchant, en principe, l'aliénation de postes de didactiques situés dans les Facultés. Notons également l'intégration des chargés d'enseignement dans les équipes de recherche qui implique une orientation de la recherche vers les pratiques d'enseignement et d'apprentissage scolaire; les nominations dans les facultés qui sont contrôlées par des représentants de l'IUFE; des projets de recherche collectifs, notamment en didactique, qui créent des synergies qui enrichissent les problématiques de chacune des équipes et constituent une force motrice.

Une chaîne reliant pratique et formation universitaire La relation entre formation dans la pratique et formation à l'université pose de délicats problèmes d'ingénierie de formation. ${ }^{10}$ Le dispositif envisage en son centre une formation où la pratique représente un volume en heures très important: importance quantitative certes, mais aussi qualitative en termes d'étendue des responsabilités puisqu'en deuxième année, les étudiants stagiaires enseignent à mi-temps, assumant les principales tâches d'un enseignant en fonction: se forgent ici des savoirs divers issus de la pratique. Ce stage en responsabilité est encadré à la fois par un formateur de terrain ${ }^{11}$ qui suit le stagiaire régulièrement dans sa classe et par un chargé d'enseignement inséré dans l'équipe 
de recherche correspondant à la didactique de la discipline du stagiaire, ce qui permet de multiples mouvements d'alternance entre formation dans la pratique et formation universitaire ${ }^{12}$, autour de trois composantes:

- la formation professionnelle pratique dans les écoles;

- la formation théorico-pratique qui articule formation sur le terrain, analyse des pratiques (didactiques et approches transversales) et recherche;

- la recherche scientifique sur les pratiques dans toutes leurs dimensions.

La première composante est assumée essentiellement par les formateurs de terrain en collaboration avec les chargés d'enseignement; la deuxième composante est prise en charge surtout par les chargés d'enseignement, tous intégrés dans des équipes de recherche; la troisième composante repose sur les professeurs et maitres d'enseignement et de recherche (MER), en étroite interaction avec les chargés d'enseignement, qui se trouvent de la sorte constituer la véritable charnière de la formation reliant la pratique avec la recherche, et, d'une certaine manière, la pratique professionnelle avec des disciplines scientifiques.

\section{Unité de recherche et de formation}

La particularité d'une haute école est d'être basée constitutivement sur le principe de l'unité de la recherche et de l'enseignement. Pour assurer ce principe au sein de la formation des enseignants du secondaire, la formation est assumée par un corps enseignant organisé en équipes de recherche, elles-mêmes animées par des professeurs $^{13}$ autour d'un triple dispositif institutionnel:

1. Tous les cours et les séminaires de recherche sont donnés par des professeurs d'université ou par des MER qui - et c'est leur mandat fondamental - mènent une activité de recherche dans le domaine de leur enseignement, garantissant ainsi un transfert continuel entre recherche et formation.

2. Les ateliers constituent un élément central de la formation professionnelle dans la mesure où y sont menées une réflexion sur et une analyse de la pratique professionnelle. Ils sont assumés par des chargés d'enseignement qui sont tous institutionnellement intégrés dans des équipes professorales et participent à la recherche à raison d'un tiers temps de leur cahier des charges. Les ateliers sont donc également nourris par des connaissances provenant de l'activité de recherche.

3. Par ailleurs, tous les étudiants participent à un séminaire de recherche dans lequel ils apprennent à connaître comment se fait la recherche en éducation en participant concrètement à des projets menés par des professeurs ou MER en charge de ces séminaires.

Les équipes de recherche intervenant dans la formation des enseignants, du primaire et du secondaire, se répartissent en deux grandes unités: les didactiques et les sciences de l'éducation. 
Tableau 1: Équipes des recherches intervenant dans la formation des enseignants du secondaire

Didactiques

- Didactique de l'éducation physique et sportive

- Didactique de la géographie

- Didactique des arts (musique et arts visuels)

- Didactique des langues étrangères

- Didactique des littératures

- Didactique des mathématiques à Genève

- Didactique des sciences (biologie)

- Didactique des sciences (physique et chimie)

- Didactique du français langue première

- Didactique de l'histoire et de la citoyenneté

\section{Sciences de l'éducation}

- Développement, apprentissage et intervention en situations scolaires

- Dimensions relationnelles et affectives de l'éducation et de la formation

- Équipe de recherche pour l'histoire (des sciences) de l'éducation

- Évaluation, régulation et différenciation des apprentissages

- Laboratoire, Innovation, Formation, Éducation

- Sociologie de l'action - Transformations des institutions - Éducation

- Relations interculturelles et formation des enseignants - Genre et éducation

- Théories, actions, langages et savoirs (en formation à l'enseignement)

Comme le montre la liste, la plupart des didactiques disciplinaires et les domaines essentiels de la pratique d'enseignement sont représentées à travers des équipes de recherche. Reste toutefois posée la question de la place de la didactique par rapport aux sciences de l'éducation: en leur sein ou à l'extérieur? Témoignant de la double insertion des didactiques des disciplines qui se réferent à la fois à des facultés qui développent des savoirs en lien avec ceux à enseigner (lettres, sciences, sciences économiques et sociales) ainsi qu'aux sciences de l'éducation, la moitié des chaires sont insérées dans les facultés, les autres en section des sciences de l'éducation. Comme l'observent Schneuwly et Hofstetter (2006), les didactiques des disciplines sont soumises à deux forces antagonistes:

- l'attirance par les sciences de référence liées aux contenus enseignés, qui signifie un retour toujours possible à l'applicationnisme;

- la centration exclusive sur les processus d'enseignement/apprentissage, indépendamment des contenus disciplinaires.

Ces forces agissent différemment pour l'enseignement primaire, qui tire plus vers la deuxième, alors que l'enseignement secondaire tend vers la première, générant une scission au sein même de la profession enseignante. Pour la formation au 
secondaire, la didactique est souvent perçue non comme un savoir spécifique, mais comme une matière essentiellement pratique; du coup, son développement paraît secondaire et fortement dépendant des disciplines de référence (Hofstetter, Schneuwly \& Lussi Borer, 2009).

\section{Conclusion: Le cas genevois mis en perspective}

La description générale d'un dispositif de formation lisse les nombreux problèmes que pose sa réalisation concrète et que seule l'étude empirique des pratiques réelles saurait mettre en évidence. Les pratiques mettant en œuvre le dispositif le transforment à leur tour, l'enrichissant tout autant qu'elles le dévient des fins visées. Dans la réalité, les articulations entre institutions, entre disciplines, entre pratiques et recherche sont loin d'être parfaites; les collaborations concrètes dans les équipes de recherches souffrent de mille imperfections, voire ne se réalisent pas du tout; la collaboration entre formateurs de terrain et chargés d'enseignement est tributaire de contextes parfois peu favorables en terme de déplacement et de temps, mais également de relations difficiles entre les personnes. On pourrait ainsi multiplier les problèmes liés à la mise en œuvre du dispositif tel que prévu dans les textes officiels. Reste néanmoins que ce dernier guide concrètement l'action de chacune des personnes participant à la formation. Il est la résultante d'une adaptation des grandes tendances internationales de réforme de la formation des enseignants dans un contexte local. Le but de la présente description était précisément de montrer, à un niveau abstrait et général, la forme que prend cette réforme et de l'expliquer par des facteurs agissant sur elle qui sont le produit de l'histoire du lieu, en l'occurrence Genève.

Adoptant comme point de vue privilégié le rapport entre profession et disciplines dans le processus incessant de professionnalisation et disciplinarisation ${ }^{14}$ à travers la formation, quatre axes forts caractérisent le processus dans le contexte genevois:

- La formation est reliée aux disciplines de référence des branches enseignées à la fois institutionnellement, par la participation des facultés à l'IUFE, et conceptuellement, à travers les didactiques qui y occupent une place centrale.

- Par la participation d'un large éventail d'équipes de recherche provenant des didactiques et des sciences de l'éducation, la formation est directement reliée à la production scientifique dans ces deux domaines.

- La formation elle-même se présente comme un dispositif pluridisciplinaire mettant en synergie des disciplines ayant trait aux savoirs à enseigner et pour enseigner, constituant des références pour la profession.

- La formation dans la pratique est articulée à la formation à l'université par le biais d'équipes réunissant des formateurs de terrain, enseignants chevronnés des branches d'enseignement, et des chargés d'enseignement, chargés 
de la réflexion et de l'analyse des pratiques d'un point de vue didactique, eux-mêmes insérés dans des équipes de recherche.

Si l'on compare les spécificités du dispositif genevois avec d'autres, on voit que la place des didactiques et l'articulation entre recherche et formation different sensiblement. A l'Université de Montréal, par exemple, les enseignements didactiques sont sous la responsabilité de la Faculté d'éducation qui est maître d'œuvre de la formation des enseignants, allant chercher au sein des facultés disciplinaires les contenus de savoirs nécessaires (Tardif \& Borges, 2009). Ce contexte imprime au dispositif un mode de fonctionnement séparé entre recherche et enseignement académique d'une part, formation professionnelle de l'autre, sans qu'existent des lieux d'articulation systématique, notamment en didactique. De manière encore différente, au sein de la Haute école pédagogique Berne-Jura-Neuchâtel (BEJUNE), la composante sciences de l'éducation et psychopédagogie joue un rôle central dans la formation théorique, la didactique étant clairement moins valorisée du point de vue de l'attribution horaire et des crédits de formation. La formation pratique, elle, est assumée par des formateurs chevronnés qui n'ont pas ou peu d'insertion académique. L'articulation entre recherche et formation n'est ainsi pas systématiquement prévue au niveau institutionnel.

A Genève, la place centrale des didactiques et l'étroite relation entre recherche et formation visent à faciliter un passage organique entre formation disciplinaire et professionnelle, donnant aux disciplines contributives une place relativement précise dans la transition entre études disciplinaires et pratique professionnelle. Investies d'un rôle médiateur, les didactiques disciplinaires ont donc pour ambition de faire dialoguer les disciplines scientifiques, le champ disciplinaire des sciences de l'éducation et le champ professionnel de l'enseignement.

Les dispositifs, tous trois inscrits dans la mouvance de réforme de la formation des enseignants qui vise à la fois une qualification élevée et une meilleure articulation entre formation théorique et pratique, sont hautement contrastés en fonction des contextes locaux. Le dispositif genevois est de ceux qui donnent aux didactiques la place la plus importante, aussi bien dans la formation pratique que théorique et qui promeut le plus puissamment ce champ de recherche et enseignement. C'est le résultat de la configuration genevoise particulière: la forte orientation disciplinaire nécessitant et rendant possible un travail concentré sur les savoirs; la nécessaire implication des facultés qui ne peut se faire que par les didactiques; une habitude de collaboration des didactiques dans et avec les sciences de l'éducation. Il s'agit maintenant d'observer concrètement les pratiques qui s’y déploient. Place donc à la recherche empirique...

\section{Notes}

1 Nous remercions Valérie Lussi Borer, co-éditrice du volume, pour sa précieuse aide aboutissant à une substantielle amélioration de la première version. L'un des experts de la deuxième version nous a rendu attentif à des problèmes subsistants quant à certains concepts, à des questions de point de vue énonciatif et à des incompréhensions que 
pouvaient susciter certaines formulations; nous avons essayé de tenir compte de ses remarques. Qu'il soit ici aussi remercié.

2 Les mouvements internationaux sont prédominants en matière de formation des enseignants comme le montre par exemple Beech (2011) en retraçant minutieusement la préparation des réformes actuelles à travers les discours en large partie convergents de l'UNESCO, de la Banque mondiale et de l'OCDE.

3 Citons notamment les archives du Département de l'Instruction publique et de l'Université détaillées in Hofstetter (2010), Hofstetter et al. (2007) et Lussi Borer (2008).

4 Les documents officiels régissant la formation (règlements, plans d'études, horaires) se trouvent sur le site http://www.unige.ch/iufe/enseignements/formations/enseignementsecondaire.html\#12; de nombreux autres documents présentant la formation se trouvent sur le site http://www.unige.ch/iufe/institut.html. Dans le présent texte, nous nous référons en outre à une série de documents, énumérés en fin de texte sous le titre «Sources». Deux des auteurs du présent texte ont été des acteurs essentiels de la réforme et ont rédigé une partie des textes énumérés dans les sources. Nous sommes bien conscients du problème que peut poser le fait d'être auteurs des prescriptions que nous présentons et analysons ici. L'objectif visé dans le présent texte ne nous semble pas pour autant contradictoire avec ce double statut.

5 Relevons que la formation des enseignants du primaire est déjà confiée dans sa totalité à l'Université depuis 1996 et qu'au moment de cette universitarisation, le parlement a émis le vœu d'une réunion des formations d'enseignants au sein de l'université.

6 Il existe par ailleurs des conventions avec les Hautes écoles de musique (HEM) et d'art et design (HEAD) qui prévoient des collaborations dans leurs domaines de spécialité.

7 Il est important de souligner que les savoirs à enseigner sont le produit complexe de multiples processus de transposition dont l'étude constitue l'un des objets essentiels de recherche en didactique. Comme le montrent ces recherches, ces savoirs n'ont pas leur seule origine dans les disciplines académiques de référence des branches ou disciplines scolaires.

8 Nous n'entrons pas ici en matière sur le rapport complexe avec les savoirs d'expérience, objet plus particulièrement lié à la formation à la pratique; voir prochain sous-chapitre.

9 Encore que des aménagements en fonction de la profession enseignante existent dans toutes les facultés avec des effets sur les contenus enseignés, voire parfois, sur la recherche.

10 On notera que nous ne la désignons pas par l'association usuelle de concepts, le rapport entre théorie et pratique, qui laisse supposer que la théorie est absente dans la pratique, et la pratique absente dans la théorie; une telle dichotomie empêche d'emblée de mettre ces deux termes en interaction, les oppose comme deux entités qui n'auraient rien à voir l'une avec l'autre.

11 Les formateurs de terrain sont des enseignants déchargés pour une ou plusieurs heures afin de suivre les stagiaires en responsabilité sur le terrain, en étroite collaboration avec les chargés d'enseignement universitaires. L'évaluation des étudiants en stage en responsabilité se fait de manière commune à travers des rencontres tripartites avec les étudiants.

12 Relevons que ce stage est accompagné d'un stage en accompagnement dans l'autre niveau d'enseignement (secondaire I pour ceux qui sont en stage en responsabilité au secondaire II et inversement), sous le double encadrement d'un formateur de terrain et d'un enseignant d'accueil.

$13 \mathrm{Ou}$, exceptionnellement, des maîtres d'enseignement et de recherche (MER).

14 Nous avons montré ailleurs pourquoi nous considérons ce processus non pas comme une évolution récente, mais comme inhérent à l'apparition même de l'enseignement comme profession au 19e siècle (Schneuwly, 2012) 


\section{Sources}

Groupe de pilotage (2005). Formation des enseignants secondaires dans le contexte suisse et européen. Vers un renouvellement de la formation professionnelle initiale des enseignants $d u$ secondaire à Genève. Genève: Département de l'instruction publique.

Groupe opérationnel (2006). Formation des enseignants du secondaire dans le canton de Genève. Rapport final. Genève: Département de l'instruction publique.

Groupe de travail (2007). Institut universitaire de formation des enseignants. Rapport du groupe de travail. Université de Genève.

Groupe de travail ad hoc (2007). Dispositif de formation en alternance: stages en responsabilité d'enseignement ou stages accompagnés? Genève: Département de l'instruction publique.

Conseil d'État de Genève (2009). Rapport du Conseil d'État au Grand Conseil sur la formation initiale des enseignants primaires et secondaires. Genève: Secrétariat du Grand Conseil.

\section{Références bibliographiques}

Ambühl, H. \& Stadelmann, W. (Éd.). (2010). Tertiarisierung der Lehrerinnen- und lehrerbildung. Bern: Schweizerische Konferenz der kantonalen Erziehungsdirektoren.

Barbier, J.-M. (Éd.). (1996). Savoirs théoriques et savoirs d'action. Paris: PUF.

Beech, J. (2011). Global panceas, local realities. international agencies and the future of education. Berne: Lang.

Berthoud, C. (2006). Une école du second degré "élitaire» pour tous ou le péril de l'école moyenne: Une histoire du secondaire obligatoire à Genève (1927-1977). Thèse de doctorat en Lettres, Université de Genève.

Blanckaert, C., Blondiaux, L., Loty, L., Renneville, M. \& Richard, N. (Éd.). (1999). L'histoire des sciences de l'homme: Trajectoire, enjeux et questions vives. Paris: L'Harmattan.

Bourdieu, P. (2001). Science de la science et réflexivité. Paris: Raisons d'agir.

Bourdoncle, R. (1991). La professionnalisation des enseignants. Analyses anglaises et américaines. 1. La fascination des professions. Revue Française de Pédagogie 94, 73-92.

Bourdoncle, R. (1993). La professionnalisation des enseignants. Analyses anglaises et américaines. 2. Les limites d'un mythe. Revue Française de Pédagogie, 105, 83-119.

Chevallard, Y. (1997). Les savoirs enseignés et leurs formes scolaires de transmission: Un point de vue didactique. Skhôlé, 7, 45-64.

Criblez, L. (2010). Die Reform der Lehrerinnen- und Lehrerbildung in der Schweiz seit 1990: Reformprozesse, erste Bilanz und Desiderata. In H. Ambühl \& W. Stadelmann (Éd.), Tertiarisierung der Lehrerinnen- und Lehrerbildung (pp. 22-58). Bern: Schweizerische Konferenz der kantonalen Erziehungsdirektoren.

Extermann, B. (2007). La professionnalisation émergente des enseignants secondaires dans un champ de tensions: Étude historique d'un moment clé de l'évolution de la formation pédagogique à Genève (1975-1977). Manuscrit non publié, Université de Genève, Section des sciences de l'éducation.

Hebrard, J. (1988). Apprendre à lire à l'école en France: Un siècle de recommandations officielles. Langue française, 80, 111-128.

Hofstetter, R. (2010). Genève: Creuset des sciences de l'éducation (fin du $19 e$ siècle - première moitié du 20e siècle). Genève: Droz.

Hofstetter, R. \& Schneuwly, B. (Éd.). (2002). Science(s) de l'éducation 19e-20e siècles. Entre champs professionnels et champs disciplinaires. Erziehungswissenschaft(en) 19.-20. Jahrhundert. Zwischen Profession und Disziplin. Berne: Lang

Hofstetter, R. \& Schneuwly, B. (Éd.). (2009). Savoirs en (trans)formation (Vol. Raisons éducatives, pp. 7-40). Bruxelles: De Boeck

Hofstetter, R., Schneuwly, B., avec la collaboration de Cicchini, M., Lussi, V., Criblez, L. \& Spaeni, M. (2007). Emergence des sciences de l'éducation en Suisse, à la croisée de traditions académiques contrastées (fin du $19^{e}$-première moitié du $20^{e}$ siècle). Berne: Lang. 
Hofstetter, R., Schneuwly, B., Lussi, V. \& Cicchini, M. (2004). Formation des enseignants secondaires: Logiques disciplinaires ou professionnelles. Le cas de Genève (fin du $19^{\mathrm{e}}-$ première moitié du $20^{\mathrm{e}}$ siècle). Revue Suisse d'Histoire, 3, 275-305.

Hudson, B. \& Meinert, M. (Éd.). (2011). Beyond fragmentation. Didactics, learning and teaching. London: Sage.

Hudson, B. \& Schneuwly, B. (2007). Didactics: Learning and teaching in Europe. European Educational Research Journal, 6, 106-108.

Lussi Borer, V. (2008). Formations à l'enseignement et sciences de l'éducation. Analyse comparée des sites universitaires de Suisse romande entre la fin du $19^{e}$ et la première moitié du $20^{e}$ siècle. Thèse de doctorat en Sciences de l'éducation, Université de Genève.

Lussi, V. \& Maulini, O. (2007). L'alternance entre logiques universitaire et professionnelle: Le cas de la formation à l'enseignement primaire à Genève au 20e siècle. In F. Mehran, C. Ronveaux \& S. Vanhulle (Éd.), Alternance(s) en formation (Vol.Raisons éducatives, pp. 103-119). Bruxelles: De Boeck.

Novoa, A. (1998). Professionnalisation des enseignants et Sciences de l'éducation. In A. Novoa, Histoire \& comparaison (Essais sur l'éducation) (pp. 121-147). Lisbonne: Educa.

Pestre, D. (2006). Introduction aux «Science Studies». Paris: La Découverte.

Schneuwly, B. \& Hofstetter, R. (2006). Les didactiques des disciplines: Entre logiques disciplinaire et professionnelle. Réflexion à partir de l'histoire de la formation des enseignants du secondaire à Genève. Bulletin de l'Association suisse des professeurs d'université (APU), 32, (1), 16-20.

Schneuwly, B. (2008). Institutionnalisation universitaire de la didactique (de la langue première): L'exemple de la suisse (et une excursion en Allemagne). La Lettre de l'AIRDF, 42, $13-15$.

Schneuwly, B. (2011). Subject didactics - an academic field related to teacher profession and teacher éducation. In B. Hudson \& M. Meinert (Éd.), Beyond fragmentation (pp. 275-286). London: Sage.

Schneuwly, B. (2012). Didactique des disciplines et profession des enseignants: Transformations récentes d'un duo ancien de la professionnalisation. In M. Mellouki \& B. Wentzel (Éd.), Que faut-il penser de la professionnalisation de la formation des enseignants aujourd'hui? (pp. 29-51). Presses Universitaires de Nancy.

Schriewer, J. (2001). Fortschrittsmodelle und Modellkonstruktionen. Formen der Internationalisierung pädagogischen Wissens. In J. Büschefeld, H. Franz \& F.-M. Kuhlemann (Éd.), Wissenschaftsgeschichte heute. Festschrift für Peter Lundgreen (pp. 302-327). Bielefeld: Verlag für Regionalgeschichte.

Tardif, M. \& Borges, C. (2009). L'internationalisation de la professionnalisation de la formation à l'enseignement secondaire et ses retraductions dans des formes sociales nationales. In R. Hofstetter \& B. Schneuwly (Éd.), Savoirs en (trans)formation. Au cour des professions de l'enseignement et de la formation (Vol. Raisons éducatives, pp. 109-136). Bruxelles: De Boeck.

Thévenaz, Th. \& Sieber, P. (2005). Didactique(s) - entre continuité et réorientation. Revue suisse des sciences de l'éducation, 27. (Éds.)

Tenorth, H.-E. (1999). Der Beitrag der Erziehungswissenschaft zur Professionalisierung pädagogischer Berufe. In H.-J. Apel, K.-P. Horn, P. Lundgreen \& U. Sandfuchs (Éd.), Professionalisierung pädagogischer Berufe im historischen Prozess (pp. 429-461). Bad Heilbrunn: Klinkhardt.

Mots-clés: Didactiques des disciplines, formation des enseignants, types de savoirs, International/local 


\section{Fachdidaktiken im Zentrum - Die Genfer Sekundar- und Mittelschullehrerausbildung}

\section{Zusammenfassung}

Der Beitrag beschreibt und analysiert eines der vielen in der Schweiz existierenden Modelle der Sekundar- und Gymnasiallehrerausbildung, nämlich dasjenige, das vor wenigen Jahren in Genf eingeführt wurde und das der Fachdidaktik einen zentralen Platz einräumt. Die Gründe und die Hypothesen, die dem Modell zugrunde liegen, werden aus drei komplementären Blickwinkeln diskutiert. Ein erster Zugang setzt bei spezifischen Faktoren des Genfer Kontexts an und untersucht, wie nationale und internationale Reformtendenzen in der Lehrerbildung an diese lokalen Gegebenheiten angepasst werden. Eine zweite Perspektive richtet sich auf verschiedene Wissenstypen, die in der Lehrerausbildung auf spezifische Art vermittelt und artikuliert werden. Es wird insbesondere der Bezug fachdidaktischen Wissens zu Wissen in Fachwissenschaft, Erziehungswissenschaft und Wissen aus professioneller Praxis analysiert. Ein dritter Fokus gilt dem Umstand, dass sich in einem Ausbildungsmodell, das universitär und interfakultär ist und das wissenschaftliche Forschung mit der Ausbildung von Lehrpersonen in Beziehung setzt, notwendigerweise die Frage stellt, wo und wie die Fachdidaktiken institutionell verankert sind.

Schlagworte: Fachdidaktiken, Lehrerbildung, Wissenstypen, international/lokal

\section{Le didattiche disciplinari al centro: la formazione degli insegnanti del secondario I a Ginevra}

\section{Riassunto}

Tra i molteplici dispositivi di formazione all'insegnamento secondario che convivono nella Confederazione svizzera, questo articolo presenta e interroga quello recentemente stabilito nel cantone di Ginevra, che conferisce un posto centrale alle didattiche disciplinari. I motivi e le ipotesi che sono alla base di questa «scommessa» delle didattiche sono interrogati da tre punti di vista complementari: il primo s'interessa al contesto ginevrino, cercando di identificare gli effetti del contesto locale sull'adattamento dei movimenti di riforma a livello nazionale e internazionale. Il secondo analizza la natura dei saperi insegnati durante la formazione concentrandosi sull'articolazione delle didattiche disciplinari con i saperi scientifici, con i saperi delle scienze dell'educazione e con i saperi della pratica professionale. Infine, il terzo considera le didattiche all'interno di in un dispositivo di formazione universitaria interfacoltà, che articola ricerca scientifica e formazione all'insegnamento.

Parole chiave: Didattiche disciplinari, formazione dei insegnanti, tipi di sapere, internazionale/locale 


\section{Subject didactics in the center - The Genevan Secondary Teacher Education}

\section{Summary}

The paper describes and analyzes one of the many existing models of secondary teacher education in Switzerland. The Genevan model was introduced some years ago; it assigns subject didactics a central role. The reasons and hypothesis underlying this choice are discussed from three points of view. 1. As everywhere, specific factors of the Genevan context transform general reform tendencies in teacher education in order to adapt them to the local context. The paper describes how this takes place. 2. Different types of knowledge are articulated in teacher education. Given the central place of didactic knowledge, its articulation with knowledge in academic reference disciplines, knowledge in educational sciences and professional knowledge is analyzed. 3. Contrary to other models in Switzerland, the Genevan teacher education is embedded in the university and is supported by several faculties. The consequences of this specificity for the institutional place of subject didactics are being discussed.

Keywords: Subject didactics, teacher education, knowledge types, international/ local 
